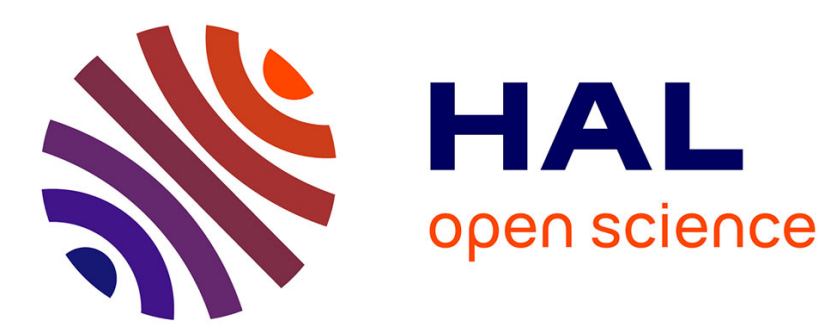

\title{
Control co-design for discrete-time switched linear systems
}

Mirko Fiacchini, Sophie Tarbouriech

\section{To cite this version:}

Mirko Fiacchini, Sophie Tarbouriech. Control co-design for discrete-time switched linear systems. Automatica, 2017, 82, pp.181-186. 10.1016/j.automatica.2017.04.043 . hal-01638569

\section{HAL Id: hal-01638569 https://hal.science/hal-01638569}

Submitted on 20 Nov 2017

HAL is a multi-disciplinary open access archive for the deposit and dissemination of scientific research documents, whether they are published or not. The documents may come from teaching and research institutions in France or abroad, or from public or private research centers.
L'archive ouverte pluridisciplinaire HAL, est destinée au dépôt et à la diffusion de documents scientifiques de niveau recherche, publiés ou non, émanant des établissements d'enseignement et de recherche français ou étrangers, des laboratoires publics ou privés. 


\title{
Control co-design for discrete-time switched linear systems *
}

\author{
Mirko Fiacchini ${ }^{\mathrm{a}}$, Sophie Tarbouriech ${ }^{\mathrm{b}}$ \\ ${ }^{a}$ Univ. Grenoble Alpes, CNRS, Gipsa-lab, F-38000 Grenoble, France \\ ${ }^{\mathrm{b}}$ LAAS-CNRS, Université de Toulouse, CNRS, Toulouse, France
}

\begin{abstract}
The paper deals with the co-design of a control policy, composed by both the state feedback and the switching control law, for discretetime switched linear systems. Constructive conditions are given that are necessary and sufficient for the stabilizability of systems which are periodic stabilizable. The conditions are in form of a Linear Matrix Inequality (LMI) problem whose solution provides the switching law and a family of state feedback gains stabilizing the system as well as a bound on the exponential decreasing rate. The effectiveness of the proposed technique is illustrated by comparison with results from the literature.
\end{abstract}

Key words: Switched linear systems; stabilizability; control co-design.

\section{Introduction}

Switched systems are characterized by a dynamics that changes with time among a finite number of different modes [12]. Switched systems attracted a notable research interest due, on the one hand, to their capability of modeling complex real systems, such as networked and embedded systems, and on the other hand to their dynamical properties, non-trivial to analyse and to design $[12,16]$.

Stability and stabilizability are central issues of the literature on switched systems, see [16] and the survey [13]. Many results are available for the problem of stability of autonomous switched systems with arbitrary switching law, like the joint spectral radius analysis [11], and the necessary and sufficient conditions given in [14]. The latter work in particular assessed that the existence of polyhedral, hence convex, Lyapunov functions is necessary and sufficient for the stability. On the other hand convex functions are proved to be conservative for switched systems with switching law as control input, see [2]. In this context many results are based on the min-switching policy, see [12], that leads to nonconvex control Lyapunov functions that are minimum of quadratics. Such functions are obtained as solutions to LMI conditions in [4], to Lyapunov-Metzler BMI conditions in $[9,10]$ and

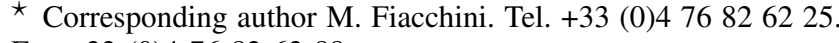
Fax +33 (0)4 76826388 .

This work has been supported by the ANR projects COMPACS (ANR-13-BS03-0004) and LimICoS (ANR-12-BS03-005-01).

Email addresses: mirko.fiacchini@gipsa-lab.fr (Mirko Fiacchini), tarbourelaas .fr (Sophie Tarbouriech).
}

through an LQR iterative procedure in [16]. The latter also proved that the existence of a minimum quadratic Lyapunov function is necessary and sufficient for stabilizability. Another necessary and sufficient condition, based in set-theory, appeared in [8]. Some of the cited conditions and novel LMI ones are analyzed and compared in [7]. The problem of codesigning both the switching law and the control input, is even more involved than the problem of stabilizability of autonomous switched systems. This kind of problem has been addressed in several works. Some approaches consist in fixing the complexity of the Lyapunov function candidates and of the control policy in function of the number of modes, as in [4,5] and [6]. Techniques based on approximating the LQR control are presented in $[17,18,1]$.

This paper deals with the co-design of the switching law and the feedback control for non-autonomous switched linear systems. The results are based on the convex conditions for stabilization of autonomous systems presented in [7], that are necessary and sufficient for periodic stabilizable systems. The problem is treated by providing an analogous LMI condition for stabilizability that is proved to be necessary and sufficient for systems that are periodic stabilizable through co-design. The LMI condition is constructive and its solution provides the control policy. The main limitation of the approach lies in its complexity that depends on the number of sequences of modes, which grows combinatorially with their maximal length considered. The method is compared with the approach presented in $[17,18]$ and with Lyapunov-Metzler approach.

Notation: Given $n \in \mathbb{N}$, define $\mathbb{N}_{n}=\{j \in \mathbb{N}: 1 \leq j \leq n\}$. 
The Euclidean-norm in $\mathbb{R}^{n}$ is $\|x\|$. The $i$-th element of a finite set of matrices is denoted as $A_{i}$. The set of $q$ switching modes is $\mathscr{I}=\mathbb{N}_{q}$, all the possible sequences of modes of length $N$ is $\mathscr{I}^{N}=\prod_{j=1}^{N} \mathscr{I}$, and $|\sigma|=N$ if $\sigma \in \mathscr{I}^{N}$. Given $N \in \mathbb{N}, N_{\mathscr{I}}=\sum_{k=1}^{N} q^{k}$ is the number of elements in $\mathscr{I}^{[1: N]}$. Given $\sigma \in \mathscr{I}^{N}$, define: $\mathbb{A}_{\sigma}=\prod_{j=1}^{N} A_{\sigma_{j}}=A_{\sigma_{N}} \cdots A_{\sigma_{1}}$, and define $\prod_{j=M}^{N} A_{\sigma_{j}}=I$ if $M>N$. Given $a \in \mathbb{R}$, the maximal integer smaller than or equal to $a$ is $\lfloor a\rfloor$.

\section{Preliminaries and problem formulation}

Consider the discrete-time switched linear system

$$
x_{k+1}=A_{\sigma_{k}} x_{k}+B_{\sigma_{k}} u_{k},
$$

where $x_{k} \in \mathbb{R}^{n}$ and $u_{k} \in \mathbb{R}^{m}$ are the state and the control input at time $k \in \mathbb{N}$, respectively; $\sigma: \mathbb{N} \rightarrow \mathscr{I}$ is the switching law and $\left\{A_{i}\right\}_{i \in \mathscr{I}}$ and $\left\{B_{i}\right\}_{i \in \mathscr{I}}$, with $A_{i} \in \mathbb{R}^{n \times n}$ and $B_{i} \in \mathbb{R}^{n \times m}$ for all $i \in \mathscr{I}$. A time-varying control policy $v: \mathbb{R}^{n} \times \mathbb{N} \rightarrow$ $\mathscr{I} \times \mathbb{R}^{m \times n}$, is such that $v(x, k)=(\sigma(x, k), K(x, k)) \in \mathscr{I} \times$ $\mathbb{R}^{m \times n}$, where $K(x, k)$ is the state feedback gain, i.e. such that $u_{k}\left(x_{k}\right)=K\left(x_{k}, k\right) x_{k}$ and then the feedback law may change at every instant.

Remark 1 As proved in [17], see Theorems 5 and 7 in particular, the attention can be restricted without loss of generality to static control policies of the form

$$
v(x)=(\sigma(x), K(x)) \in \mathscr{I} \times \mathbb{R}^{m \times n}
$$

such that $v(a x)=v(x)$ for all $x \in \mathbb{R}^{n}$ and $a \in \mathbb{R}$, and to piecewise quadratic Lyapunov functions. Moreover $K(x)$ belongs to a finite set i.e. $K(x) \in \mathscr{K}=\left\{\kappa_{i}\right\}_{i \in \mathbb{N}_{M}}$, with $M \in \mathbb{N}$.

The switched system in closed loop with (2) reads

$$
x_{k+1}=\left(A_{\sigma\left(x_{k}\right)}+B_{\sigma\left(x_{k}\right)} K\left(x_{k}\right)\right) x_{k},
$$

where $\sigma\left(x_{k}\right)=\sigma_{k}$. We denote with $x_{k}^{v}\left(x_{0}\right) \in \mathbb{R}^{n}$ the state of the system (1) at time $k$ starting from $x(0)=x_{0}$ by applying the control policy $v$. Given $\sigma \in \mathscr{I}^{D}$ we denote with $x_{k}^{\sigma}\left(x_{0}\right)$ the state of (3) at time $k \leq D$ starting at $x_{0}$ under the switching sequence $\sigma$. The dependence of $x_{k}^{v}$ and $x_{k}^{\sigma}$ on the initial conditions will be dropped when clear from the context.

Definition 1 The system (1) is globally exponentially stabilizable if there are a control policy $v(x)$ as in (2), $c \geq 0$ and $\lambda \in[0,1)$ such that $\left\|x_{k}^{v}\left(x_{0}\right)\right\| \leq c \lambda^{k}\left\|x_{0}\right\|$, for all $x_{0} \in \mathbb{R}^{n}$, with $x_{k}$ state of (3).

Some recent results from [7] concerning the stabilizability of autonomous switched linear systems $x_{k+1}=A_{\sigma_{k}} x_{k}$, with $\sigma_{k} \in \mathscr{I}$, are recalled hereafter since widely employed in the following. A periodic switching law for the system $x_{k+1}=$ $A_{\sigma_{k}} x_{k}$ is given by $\sigma(k)=i_{p(k)}$ and $p(k)=k-D\lfloor k / D\rfloor+1$, with $D \in \mathbb{N}$ and $i \in \mathscr{I}^{D}$, which means that the sequence of modes given by $i$ repeats cyclically in time.

Definition 2 The system $x_{k+1}=A_{\sigma_{k}} x_{k}$ is periodic $\sigma$ stabilizable if there exist a periodic switching law $\sigma: \mathbb{N} \rightarrow \mathbb{N}_{q}, c \geq 0$ and $\lambda \in[0,1)$ such that $\left\|x_{k}^{\sigma}(x)\right\| \leq c \lambda^{k}\|x\|$ holds for all $x \in \mathbb{R}^{n}$.

For periodic $\sigma$-stabilizability a periodic, state-independent stabilizing switching law must exists, whereas it could not exist for generic $\sigma$-stabilizability. One of the main results provided in [7] is a necessary and sufficient condition for periodic $\sigma$-stabilizability in form of LMI.

Theorem 1 A periodic $\sigma$-stabilizing switching law for the system (1) exists if and only if there exist $N \in \mathbb{N}$ and $\eta \in \mathbb{R}^{N_{\mathscr{I}}}$, with $\eta \geq 0$, such that $\sum_{i \in \mathscr{I}[1: N]} \eta_{i}=1$ and

$$
\sum_{i \in \mathscr{I}[1: N]} \eta_{i} \mathbb{A}_{i}^{T} \mathbb{A}_{i}<I
$$

In this paper, we are not interested in determining periodic stabilizing switching laws but on computing a statedependent control policy whenever the system admits a periodic stabilizing switching sequence.

Remark 2 The condition (4) can be used to determine if a periodic $\sigma$-stabilizing switching law exists, but such a switching law could be very poor in terms of convergence and very complex, as its length can be very high. In fact, supposing that (4) is satisfied or equivalently that there exists $\mu \in[0,1)$ such that $\sum_{i \in \mathscr{I}[1: N]} \eta_{i} \mathbb{A}_{i}^{T} \mathbb{A}_{i} \leq \mu I$, the periodic sequence length is bounded by $p N$ with $p$ such that $\mu^{p} n<1$ (see the proof of Theorem 22 in [7]), which can be very big for high values of $\mu$. Moreover, the convergence can be very slow (see examples in [7]).

Thus, if, on the one hand, periodic $\sigma$-stabilizability is more conservative than generic $\sigma$-stabilizability, on the other hand, the equivalent condition is much more computationally tractable, see Section 4. Indeed, the condition in case of periodic $\sigma$-stabilizability is an LMI in the parameter $N$ that might by much smaller than the periodic cycle length. In this paper we focus on a condition analogous to the LMI one (4) for the controlled switched system (1). The aim is to provide an LMI problem whose solution determines a stabilizing control policy (2) for periodic stabilizable systems.

\section{Switched state-dependent control policy}

The following lemma is functional for the main results presented in this paper. Its proof is based on the elimination (or projection) lemma, see [3], analogously to what done in [15]. The elimination lemma claims that there exists $X \in \mathbb{R}^{m \times m}$ 
satisfying $U^{T} X V+V^{T} X^{T} U+Z>0$ with $Z \in \mathbb{R}^{n \times n}$ symmetric, if and only if

$$
N_{u}^{T} Z N_{u}>0, \quad N_{v}^{T} Z N_{v}>0,
$$

with $N_{u}, N_{v} \in \mathbb{R}^{n \times m}$ such that $U N_{u}=0$ and $V N_{v}=0$.

Lemma 1 Given $M_{i} \in \mathbb{R}^{n \times n}$, with $i \in \mathbb{N}_{p}$, and the nonsingular matrix $P \in \mathbb{R}^{n \times n}$, the inequality

$$
\eta P^{T} M_{1}^{T} \ldots M_{p}^{T} M_{p} \ldots M_{1} P<I,
$$

with $\eta>0$, holds if and only if there exist $G_{i} \in \mathbb{R}^{n \times n}$, with $i \in \mathbb{N}_{p-1}$, such that

$$
\left[\begin{array}{cccccc}
\eta I & M_{p} G_{p-1} & \ldots & 0 & 0 & 0 \\
G_{p-1}^{T} M_{p}^{T} & G_{p-1}+G_{p-1}^{T} & \ldots & 0 & 0 & 0 \\
\ldots & \ldots & \ldots & \ldots & \ldots & \ldots \\
0 & 0 & \ldots & G_{2}+G_{2}^{T} & M_{2} G_{1} & 0 \\
0 & 0 & \ldots & G_{1}^{T} M_{2}^{T} & G_{1}+G_{1}^{T} & \eta M_{1} \\
0 & 0 & \ldots & 0 & \eta M_{1}^{T} & \left(P P^{T}\right)^{-1}
\end{array}\right]>0
$$

is satisfied.

Proof: Consider first the case $p=2$ and notice that $\eta P^{T} M_{1}^{T} M_{2}^{T} M_{2} M_{1} P<I$ and $\eta M_{2} M_{1} P P^{T} M_{1}^{T} M_{2}^{T}<I$ are equivalent. Conditions $\eta M_{2} M_{1} P P^{T} M_{1}^{T} M_{2}^{T}<I$ and $\eta>0$ are equivalent to (5) with

$$
N_{u}=\left[\begin{array}{c}
I \\
M_{2}^{T}
\end{array}\right], \quad N_{v}=\left[\begin{array}{l}
I \\
0
\end{array}\right], \quad Z=\left[\begin{array}{cc}
\beta I & 0 \\
0 & -M_{1} P P^{T} M_{1}^{T}
\end{array}\right],
$$

and $\beta=\eta^{-1}$. Applying the elimination lemma with $U=$ $\left[\begin{array}{ll}M_{2}^{T} & -I\end{array}\right]$ and $V=\left[\begin{array}{ll}0 & I\end{array}\right]$ we have that $\eta M_{2} M_{1} P P^{T} M_{1}^{T} M_{2}^{T}<$ $I$ and $\eta>0$ are equivalent to the existence of $X \in \mathbb{R}^{n \times n}$ such that

$$
\left[\begin{array}{cc}
\beta I & M_{2} X \\
X^{T} M_{2}^{T} & -X-X^{T}-M_{1} P P^{T} M_{1}^{T}
\end{array}\right]>0 .
$$

holds. Note that (7) implies that $X+X^{T}<0$. Pre- and postmultiplying by $\operatorname{diag}(-I, I)$ and multiplying by $\eta$, one obtains

$$
\left[\begin{array}{cc}
I & M_{2} G_{1} \\
G_{1}^{T} M_{2}^{T} & G_{1}+G_{1}^{T}-\eta M_{1} P P^{T} M_{1}^{T}
\end{array}\right]>0
$$

with $G_{1}=-\eta X$, which is equivalent to (6).

To extend iteratively the result for the case of $p>2$, consider first $p=3$. From what said above $\eta P^{T} M_{1}^{T} M_{2}^{T} M_{3}^{T} M_{3} M_{2} M_{1} P<$ $I$ and $\eta>0$ are equivalent, see (7), to the existence of $X$ such that

$$
\left[\begin{array}{cc}
\beta I & M_{3} X \\
X^{T} M_{3}^{T} & -X-X^{T}-M_{2} M_{1} P P^{T} M_{1}^{T} M_{2}
\end{array}\right]>0 .
$$

Condition (8) and $\eta=\beta^{-1}>0$ are equivalent to (5) with

$$
\begin{aligned}
& N_{u}=\left[\begin{array}{cc}
I & 0 \\
0 & I \\
0 & M_{2}^{T}
\end{array}\right], \quad N_{v}=\left[\begin{array}{ll}
I & 0 \\
0 & I \\
0 & 0
\end{array}\right], \quad \begin{array}{l}
U=\left[\begin{array}{lll}
0 & M_{2}^{T} & -I
\end{array}\right], \\
0
\end{array} \\
& Z=\left[\begin{array}{ccc}
\beta I & M_{3} X & 0 \\
X^{T} M_{3}^{T} & -X-X^{T} & 0 \\
0 & 0 & -M_{1} P P^{T} M_{1}^{T}
\end{array}\right]
\end{aligned}
$$

From the elimination lemma, they are also equivalent to the existence of $Y$ satisfying

$$
\left[\begin{array}{ccc}
\beta I & M_{3} X & 0 \\
X^{T} M_{3}^{T} & -X-X^{T} & M_{2} Y \\
0 & Y^{T} M_{2}^{T} & -Y-Y^{T}-M_{1} P P^{T} M_{1}^{T}
\end{array}\right]>0,
$$

which corresponds to (6) with $G_{2}=-\eta X$ and $G_{1}=-\eta Y$. Applying analogous reasonings, and using (9) as (7) for $p=3$, the result can be proven for $p=4$ and so on.

\subsection{Switching law and feedback control co-design}

From Remark 1, the problem of co-design is equivalent to determine a stabilizing static control policy as in (2), with finite number of feedback gains, and a piecewise quadratic Lyapunov function for the system (3). The first step is to apply the result in Theorem 1 for the co-design. This would lead to a non-static stabilizing control policy, as the control would result in sequences of modes and gains. Then a static control policy, as defined in Remark 1, will be determined. Applying Theorem 1, the objective is to search for sequences of modes and feedback gains, fulfilling the LMI condition (4) in the context of co-design. That is, given a sequence $\vartheta \in \mathscr{I}$, of length $J$, and a time instant $j \in \mathbb{N}_{J}$, a gain among the finite set $\mathscr{K}$ can be applied, denoted as $K_{j}^{\vartheta}$ and whose value has to be designed. Then, with a slight abuse of notation, given $J \in \mathbb{N}$ and a sequence $\vartheta \in \mathscr{I}^{J}$, we denote

$$
\mathbb{F}_{\vartheta}=\prod_{j=1}^{J} F_{\vartheta_{j}}=F_{\vartheta_{J}} \ldots F_{\vartheta_{1}}=\left(A_{\vartheta_{J}}+B_{\vartheta_{J}} K_{J}^{\vartheta}\right) \ldots\left(A_{\vartheta_{1}}+B_{\vartheta_{1}} K_{1}^{\vartheta}\right)
$$

Thus a set of $N_{\mathscr{I}}=\sum_{k=1}^{N} q^{k}$ matrices $\mathbb{F}_{\vartheta}$, one for every $\vartheta \in \mathscr{I}^{[1: N]}$, can be defined as in (10) that are parameterized

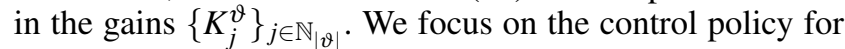
(1) of the form (2) where $K(x)$ belongs to one of the elements of a sequence associated to a mode in $\mathscr{I}[1: N]$. Then, $K(x)$ is a gain among the $\sum_{k=1}^{N} k q^{k}$ possible, i.e. $K(x) \in \mathscr{K}$ where

$$
\mathscr{K}=\left\{\kappa_{i}\right\}_{i \in \mathbb{N}_{M}}=\left\{K_{j}^{\vartheta} \in \mathbb{R}^{m \times n}: \vartheta \in \mathscr{I}^{[1: N]}, j \in \mathbb{N}_{|\vartheta|}\right\},
$$

with $M=\sum_{k=1}^{N} k q^{k}$. Given a switching law $\vartheta: \mathbb{N} \rightarrow \mathscr{I}$ and a sequence of feedback gains $K^{\vartheta}: \mathbb{N} \rightarrow \mathbb{R}^{m \times n}$, we denote 
with $x_{k}^{\vartheta}(x)$ the state at time $k$ starting at $x$ if the control $v_{k}=\left(\vartheta_{k}, K_{k}^{\vartheta}\right)$ is applied at $k$ for all $k \in \mathbb{N}$. Analogously to the case without control input, the concept of periodic $\vartheta$ stabilizability can be given for the system (1).

Definition 3 The system (1) is periodic $\vartheta$-stabilizable if there exist: a periodic switching law $\vartheta: \mathbb{N} \rightarrow \mathscr{I}$ and a periodic sequence $K^{\vartheta}: \mathbb{N} \rightarrow \mathbb{R}^{m \times n}$, both of cycle length $D \in \mathbb{N}$; $c \geq 0$ and $\lambda \in[0,1)$ such that $\left\|x_{k}^{\vartheta}(x)\right\| \leq c \lambda^{k}\|x\|$ holds for all $x \in \mathbb{R}^{n}$ and $k \in \mathbb{N}$.

Clearly periodic $\vartheta$-stabilizability is sufficient for exponential stabilizability of (1) as in Definition 1. From Definition 3 and Theorem 1, the LMI conditions

$$
\sum_{i \in \mathscr{I}[1: N]} \eta_{i}=1
$$

and

$$
\sum_{j \in \mathscr{I}[1: N]} \eta_{j} \mathbb{F}_{j}^{T} \mathbb{F}_{j}<I .
$$

are necessary and sufficient for periodic $\vartheta$-stabilizability of system (1). Thus, conditions (13) provides the exact characterization of $\vartheta$-stabilizability, together with (12). Before giving a convex condition equivalent to (13), we provide a corollary that is useful to directly apply the results of Lemma 1 , that requires strictly positive values of $\eta$.

Corollary 1 The switched system (1) is periodic $\vartheta$ stabilizable if and only if there exist $N \in \mathbb{N}$, the set of feedback gains (11) and $\eta \in \mathbb{R}^{N_{\mathscr{I}}}$ such that (12) and (13) hold with $\eta>0$.

Proof: Sufficiency follows trivially from Theorem 1, since $\eta>0$ implies $\eta \geq 0$. Suppose now that (12) and (13) hold with $\eta \geq 0$, where at least one $\eta_{j}$ is zero. Condition (13) is equivalent to the existence of $\mu>0$ such that $\sum_{j \in \mathscr{I}[1: N]} \eta_{j} \mathbb{F}_{j}^{T} \mathbb{F}_{j} \leq \mu I$. Define $J \subseteq \mathscr{I}[1: N]$ the indices such that $\eta_{j}=0$ if $j \in J$, and $\bar{J}$ its complement in $\mathscr{I}^{[1: N]}$, and denote with $|J|$ and $|J|$ their cardinality. Given $\varepsilon>0$ we have

$$
\begin{aligned}
\sum_{j \in \mathscr{I}[1: N]} \eta_{j} \mathbb{F}_{j}^{T} \mathbb{F}_{j}= & \sum_{j \in J} \frac{\varepsilon}{|J|} \mathbb{F}_{j}^{T} \mathbb{F}_{j}+\sum_{j \in \bar{J}}\left(\eta_{j}-\frac{\varepsilon}{|\bar{J}|}\right) \mathbb{F}_{j}^{T} \mathbb{F}_{j} \\
& -\sum_{j \in J} \frac{\varepsilon}{|J|} \mathbb{F}_{j}^{T} \mathbb{F}_{j}+\sum_{j \in \bar{J}} \frac{\varepsilon}{|\bar{J}|} \mathbb{F}_{j}^{T} \mathbb{F}_{j} \leq \mu I .
\end{aligned}
$$

Hence, choosing $\varepsilon>0$ such that

$$
\varepsilon\left(\sum_{j \in J} \frac{1}{|J|} \mathbb{F}_{j}^{T} \mathbb{F}_{j}-\sum_{j \in J} \frac{1}{|\bar{J}|} \mathbb{F}_{j}^{T} \mathbb{F}_{j}\right)<(1-\mu) I
$$

and $\varepsilon<|\bar{J}| \eta_{j}$ for all $j \in \bar{J}$, we have that $v \in \mathbb{R}^{N_{\mathscr{I}}}$ defined by $v_{j}=\varepsilon /|J|$ if $j \in J$, and $v_{j}=\eta_{j}-\varepsilon /|\bar{J}|$ if $j \in \bar{J}$, is such that $v>0, \sum_{j \in \mathscr{I}[1: N]} v_{j}=1$ and $\sum_{j \in \mathscr{I}[1: N]} v_{j} \mathbb{F}_{j}^{T} \mathbb{F}_{j}<I$, from (14) and (15).

Hence, from Corollary 1, the constraints on non-negativity of $\eta$ can be replaced with its positivity.

Proposition 1 Given $N \in \mathbb{N}, \eta \in \mathbb{R}^{N_{\mathscr{I}}}$ with $\eta>0$, and the set of feedback gains (11), condition (13) holds if and only if for every $j \in \mathscr{I}^{[1: N]}$ there exist $|j|-1$ nonsingular matrices $G_{j, k} \in \mathbb{R}^{n \times n}$ with $k \in \mathbb{N}_{|j|-1}$ and $R_{j} \in \mathbb{R}^{n \times n}$ such that $R_{j}=$ $R_{j}^{T}>0$ and

$$
\left[\begin{array}{ccccccc}
\eta_{j} I & X_{j,|j|} & 0 & \ldots & 0 & 0 & 0 \\
X_{j,|j|}^{T} & Y_{j,|j|-1} & X_{j,|j|-1} & \ldots & 0 & 0 & 0 \\
0 & X_{j,|j|-1}^{T} & Y_{j,|j|-1} & \ldots & 0 & 0 & 0 \\
\ldots & \ldots & \ldots & \ldots & \ldots & \ldots & \\
0 & 0 & 0 & \ldots & Y_{j, 2} & X_{j, 2} & 0 \\
0 & 0 & 0 & \ldots & X_{j, 2}^{T} & Y_{j, 1} & X_{j, 1} \\
0 & 0 & 0 & \ldots & 0 & X_{j, 1}^{T} & R_{j}
\end{array}\right]>0
$$

for every $j \in \mathscr{I}^{[1: N]}$ with

$$
\begin{array}{ll}
X_{j, 1}=\eta_{j} \mathbb{F}_{j_{1}}, & \\
X_{j, k+1}=\mathbb{F}_{j_{k+1}} G_{j, k}, & \forall k \in \mathbb{N}_{|j|-1}, \\
Y_{j, k}=G_{j, k}+G_{j, k}^{T}, & \forall k \in \mathbb{N}_{|j|-1},
\end{array}
$$

and

$$
\sum_{j \in \mathscr{I}[1: N]} R_{j}<I
$$

Proof: Condition (13) is equivalent to the existence of $P_{i} \in \mathbb{R}^{n \times n}$ such that

$$
\left(P_{i} P_{i}^{T}\right)^{-1}:=I-\sum_{j \in \mathscr{I}[1: N]} \eta_{j} \mathbb{F}_{j}^{T} \mathbb{F}_{j}>\eta_{i} \mathbb{F}_{i_{1}}^{T} \ldots \mathbb{F}_{i_{|i|}}^{T} \mathbb{F}_{i_{|i|}} \ldots \mathbb{F}_{i_{1}} \geq 0,
$$

for every $i \in \mathscr{I}^{[1: N]}$, hence with $P_{i}$ nonsingular. Then (19) is equivalent to $I>\eta_{i} P^{T} \mathbb{F}_{i_{1}}^{T} \ldots \mathbb{F}_{i_{|i|}}^{T} \mathbb{F}_{i_{|i|} \mid} \ldots \mathbb{F}_{i_{1}} P$ and thus, from Lemma 1 , to the existence of matrices $G_{i, k} \in \mathbb{R}^{n \times n}$, with $k \in \mathbb{N}_{|j|-1}$, and $R_{i}=R_{i}^{T}>0$ such that (16) holds with $j=i$ and $\left(P_{i} P_{i}^{T}\right)^{-1}>R_{i}$.

Condition $\left(P_{i} P_{i}^{T}\right)^{-1}=I-\sum_{j \in \mathscr{I}[1: N] \backslash i} \eta_{j} \mathbb{F}_{j}^{T} \mathbb{F}_{j}>R_{i}$ is equivalent to $\left(P_{k} P_{k}^{T}\right)^{-1}:=I-R_{i}-\sum_{j \in \mathscr{I}[1: N]} \eta_{j i, k\}} \mathbb{F}_{j}^{T} \mathbb{F}_{j}>\eta_{k} \mathbb{F}_{k_{1}}^{T} \ldots \mathbb{F}_{k_{|k|}}^{T} \mathbb{F}_{k_{|k|}} \ldots \mathbb{F}_{k_{1}}$

for any $k \in \mathscr{I}^{[1: N]} \backslash i$, with $P_{k} \in \mathbb{R}^{n \times n}$ nonsingular. From what proved above, (20) is equivalent to $I>$ 
$\eta_{k} P_{k}^{T} \mathbb{F}_{k_{1}}^{T} \ldots \mathbb{F}_{k_{|k|}}^{T} \mathbb{F}_{k_{|k|}} \ldots \mathbb{F}_{k_{1}} P_{k}$, and then also to (16) with $j=k$ and $\left(P_{k} P_{k}^{T}\right)^{-1}>R_{k}$. Repeating the reasoning for every $j \in \mathscr{I}^{[1: N]}$, condition (13) holds if and only if (16) and (18) are satisfied.

The main results of the paper are stated in the following theorems, based on Proposition 1. They provide a necessary and sufficient LMI condition for the $\vartheta$-stabilizability of switched systems (1). Moreover, the explicit form of the control law (2) is given.

Theorem 2 The switched system (1) is periodically $\vartheta$ stabilizable if and only if there exist $N \in \mathbb{N} ; \eta \in \mathbb{R}^{N_{\mathscr{I}}}$ such that $\eta>0$ and (12) holds; and for every $j \in \mathscr{I}^{[1: N]}$ there are:

- $|j|-1$ nonsingular matrices $G_{j, k} \in \mathbb{R}^{n \times n}$, with $k \in \mathbb{N}_{|j|-1}$;

- $|j|$ matrices $Z_{j, k} \in \mathbb{R}^{m \times n}$ with $k \in \mathbb{N}_{|j|}$;

- a symmetric positive definite matrix $R_{j} \in \mathbb{R}^{n \times n}$;

such that (16) and (18) hold with

$$
\begin{array}{ll}
X_{j, 1}=\eta_{j} A_{j_{1}}+B_{j_{1}} Z_{j, 1}, & \\
X_{j, k+1}=A_{j_{k+1}} G_{j, k}+B_{j_{k+1}} Z_{j, k+1}, & \forall k \in \mathbb{N}_{|j|-1}, \\
Y_{j, k}=G_{j, k}+G_{j, k}^{T}, & \forall k \in \mathbb{N}_{|j|-1},
\end{array}
$$

and feedback gains

$$
\begin{aligned}
& K_{1}^{j}=\eta_{j}^{-1} Z_{j, 1}, \\
& K_{k+1}^{j}=Z_{j, k+1} G_{j, k}^{-1}, \quad \forall k \in \mathbb{N}_{|j|-1},
\end{aligned}
$$

for every $j \in \mathscr{I}^{[1: N]}$.

Proof: From Proposition 1, (16)-(18) and (21) with (22) are equivalent to (13), which, together with (12), is necessary and sufficient for periodic $\vartheta$-stabilizability.

Any solution of the conditions of Theorem 2, besides proving $\vartheta$-stabilizability, provides a stabilizing control policy and a bound on the decreasing of the Euclidean norm every $N$ steps at most, as summarized in the following theorem.

Theorem 3 Suppose there exist $\alpha>1$ and $N \in \mathbb{N} ; \eta \in \mathbb{R}^{N_{\mathscr{I}}}$ such that $\eta>0$; matrices $G_{j, k} \in \mathbb{R}^{n \times n}$ with $k \in \mathbb{N}_{|j|-1}, Z_{j, k} \in$ $\mathbb{R}^{m \times n}$ with $k \in \mathbb{N}_{|j|}$ and $R_{j} \in \mathbb{R}^{n \times n}$ as defined in Theorem 2 such that (16)-(18) and (21) hold and

$$
\sum_{i \in \mathscr{I}[1: N]} \eta_{i}=\alpha
$$

Then system (1) is periodically $\vartheta$-stabilizable and $\left\|\mathbb{F}_{\vartheta(x)} x\right\|_{2}<\lambda\|x\|_{2}$ holds for all $x \in \mathbb{R}^{n}$, with

$$
\vartheta=\vartheta(x)=\arg \min _{j \in \mathscr{I}[1: N]}\left(x^{T} \mathbb{F}_{j}^{T} \mathbb{F}_{j} x\right)
$$

and $\lambda=\alpha^{-1 / 2}$. Given $x(t)=x$, the stabilizing control policy is defined from (22) within an horizon of length $|\vartheta|$ as

$$
v(x, k)=(\sigma(x, k), K(x, k))=\left(\vartheta_{k}, K_{k}^{\vartheta}\right)
$$

to be applied at time $t+k-1$, for all $k \in \mathbb{N}_{|\vartheta|}$.

Proof: Consider $\eta>0$ satisfying (23) and define $v=$ $\alpha^{-1} \eta$. Then, since it has been proved that the satisfaction of (16)-(18) and (21) is equivalent to (13), it follows that $\sum_{i \in \mathscr{I}^{[1: N]}} v_{i}=1$ and

$$
\sum_{j \in \mathscr{I}[1: N]} v_{j} \mathbb{F}_{j}^{T} \mathbb{F}_{j}<\alpha^{-1} I=\lambda^{2} I
$$

hold and then

$$
x^{T} \mathbb{F}_{\vartheta}^{T} \mathbb{F}_{\vartheta} x=\sum_{j \in \mathscr{I}[1: N]} v_{j} x^{T} \mathbb{F}_{\vartheta}^{T} \mathbb{F}_{\vartheta} x \leq \sum_{j \in \mathscr{I}[1: N]} v_{j} x^{T} \mathbb{F}_{j}^{T} \mathbb{F}_{j} x<\lambda^{2} x^{T} x
$$

Hence applying the control related to the sequence $\vartheta$, that is the control (25) defined for the next $|\vartheta|$ instants, leads to $\left\|\mathbb{F}_{\vartheta(x)} x\right\|_{2}<\lambda\|x\|_{2}$. The periodic $\vartheta$-stabilizability follows from Theorem 2 .

From Theorem 3, the value of $\alpha$, is related to $\lambda$ and then could serve for obtaining the fastest decreasing rate, for a given $N$, by solving the following single LMI problem

$$
\begin{array}{r}
\alpha=\sup _{\alpha, \eta, G_{j, k}, Z_{j, k}, R_{j}} \sum_{j \in \mathscr{I}[1: N]} \eta_{j} \\
\text { s.t. }(16)-(18)-(21),
\end{array}
$$

with $\eta, G_{j, k}, Z_{j, k}, R_{j}$ as defined in Theorem 2 .

Remark 3 A nonconvex control Lyapunov function $V(x)$, decreasing at every step, and a state-dependent control policy $v(x)$ as in (2) can be defined as in [7] from the solution of the LMI problem:

$$
V(x)=\min _{j \in \mathscr{O}_{N}}\left(x^{T} \lambda^{-|j|} \mathbb{F}_{j}^{T} \mathbb{F}_{j} x\right),
$$

where $\mathscr{Y}_{N}$ is the set of all suffixes of the elements of $\mathscr{I}^{[1: N]}$, and the control policy is $v(x)=\left(\hat{j}_{1}(x), K_{1}^{\hat{j}(x)}\right)$ with

$$
\hat{j}(x)=\arg \min _{j \in \mathscr{Y}_{N}}\left(x^{T} \lambda^{-|j|} \mathbb{F}_{j}^{T} \mathbb{F}_{j} x\right) .
$$

\section{Comparisons}

In this section we compare our approach with results from the literature, in terms of conservatism and complexity. 


\subsection{Comparison with switched LQR method}

As a term of comparison for our method, we consider the nice results presented in $[17,18]$ that is substantially based on the fact that a time-varying system is exponentially stabilizable if and only if the infinite-horizon LQR problem leads to a value function that is a control Lyapunov function. The method is based on a Riccati-like equation iteratively applied to generate an increasing set of gains and positive definite matrices that eventually provide the stabilizing LQR control and the related Lyapunov function. One main limitation is that the number of matrices generated might grow exponentially with the iterations, despite the criterion applied for reducing the redundancy is applied to limit the phenomenon. On the other hand, such a redundancy test would entail additional computational burden to the algorithm, already exponentially complex. Finally, a stop condition, in form of contraction test, must be checked at every iteration. Since the general condition could be overly complex, an only sufficient alternative, analogous to the one given in Theorem 1, is employed in the relaxed version of the algorithm.

Comparing our result with the LQR-based ones, the most relevant feature is the fact that in our approach the feedback gains are design variables and are effectively computed by solving the LMI problem. That is, roughly speaking, while the set of gains in the LQR approach is exponentially increased through the iterations until a stabilizing control policy is achieved, in our method the sets of gains that maximizes the contraction, for a given horizon, are directly obtained. The benefits of this co-design approach are evident in the example below, taken from $[17,18]$. Moreover, also from the computational point of view, we think that our method presents some benefits. Indeed, in spite of solving an exponentially increasing number of Riccati equations and convex optimization problems to reduce the redundancy, a single LMI problem has to be solved in our approach. Furthermore, our approach does not required a numerical search in the parameter space to obtain a solution. Finally, also from the point of view of the generality of the result, our approach is at least as general as the one proposed in $[17,18]$. In fact, the efficient algorithm stop condition is analogous to the LMI one given in Theorem 1, and then affected by the same conservatism of our approach with respect to the general stabilizability property. This means that every LQR-like solution can be recovered as a solution of our approach.

\subsection{Comparison with Lyapunov-Metzler-like conditions}

The methods based on Lyapunov-Metzler conditions, as $[9,5,6]$, seem to provide more conservative results, as the complexity of the Lyapunov function and control policy are fixed in function of the modes number. The conservatism is proved in the following example based on Example 27 in [7], used to prove analogous results for autonomous switched systems.
Example 1 Given $n=3$ and $q=2$, define

$$
A_{1}=\left[\begin{array}{ccc}
1 & 0 & 0 \\
0 & 0.5 & 0 \\
0 & 0 & a
\end{array}\right], A_{2}=\left[\begin{array}{ccc}
4 & -4 & 0 \\
4 & 4 & 0 \\
0 & 0 & a
\end{array}\right], B_{1}=B_{2}=\left[\begin{array}{l}
0 \\
0 \\
1
\end{array}\right]
$$

Clearly the subsystem $x_{3}$ is stabilized by $u=K x_{3}$ with $|a+K|<1$. The subsystem $\left(x_{1}, x_{2}\right)$ is stabilizable through an appropriate switching sequence obtained by solving the LMI condition (4) but does not admit any solution to the Lyapunov-Metzler condition, see [7].

From the computational point of view, if on one side the Lyapunov-Metzler conditions are non-convex, in form of $\mathrm{BMI}$, on the other one they involve a fixed number of matrices and then do not incur in the combinatorial complexity growth that affects the method present here and in $[17,18]$.

\section{Numerical examples}

Example 2 Consider Example 2 in [17], that is the 4dimensional system with 4 modes whose matrices are

$$
\begin{aligned}
A_{1}= & {\left[\begin{array}{cccc}
0.5 & -1 & 2 & 3 \\
0 & -0.5 & 2 & 4 \\
0 & -1 & 2.5 & 2 \\
0 & 0 & 0 & 1.5
\end{array}\right], A_{2}=\left[\begin{array}{cccc}
-0.5 & -1 & 2 & 1 \\
0 & 1.5 & -2 & 0 \\
0 & 0 & 0.5 & 0 \\
-2 & -1 & 2 & 2.5
\end{array}\right], } \\
A_{3} & =\left[\begin{array}{cccc}
1.5 & 0 & 0 & 0 \\
1 & 1 & 0.5 & -0.5 \\
0 & 0.5 & 1 & -0.5 \\
1 & 0 & 0 & 0.5
\end{array}\right], A_{4}=\left[\begin{array}{cccc}
0.5 & 1 & 0 & 0 \\
0 & 0.5 & 0 & 0 \\
0 & 0 & 0.5 & 0 \\
0 & 2 & -2 & 0.5
\end{array}\right], \\
B_{1}= & {\left[\begin{array}{l}
1 \\
2 \\
3 \\
4
\end{array}\right], \quad B_{2}=\left[\begin{array}{l}
3 \\
2 \\
1
\end{array}\right], B_{3}=\left[\begin{array}{l}
4 \\
3 \\
2 \\
1
\end{array}\right], \quad B_{4}=\left[\begin{array}{l}
1 \\
2 \\
3 \\
4
\end{array}\right] . }
\end{aligned}
$$

The conditions of Theorem 3 are satisfied with horizon $N=3$. Besides the inherent computational benefit of having a stabilization condition in form of LMI with respect to the algorithmic method presented in [17], also the control obtained is substantially simpler and more efficient. Actually, in [17] stabilizability is proved by means of an algorithm which inspects control horizons of length 7 resulting in a piecewise quadratic function determined by 13 matrices. In our case, after solving the LMI problem (26), we obtain $\alpha=1145.2$, that implies $\lambda=0.0296$. The values of $\eta_{j}$ are consistently higher for two particular sequences with 
respect to the others. Indeed, the control related to the sequence $i=\{4,2,2\}$ with gains

$$
\begin{aligned}
K_{1}^{\{4,2,2\}} & =\left[\begin{array}{llll}
0.0285 & 0.0333 & -0.0715 & -0.0333
\end{array}\right], \\
K_{2}^{\{4,2,2\}} & =\left[\begin{array}{llll}
-0.8215 & -0.4668 & 0.9070 & 0.8719
\end{array}\right], \\
K_{3}^{\{4,2,2\}} & =\left[\begin{array}{llll}
0.1333 & 0.0167 & -0.1667 & -0.2167
\end{array}\right]
\end{aligned}
$$

leads to a Schur-Cohn matrix whose spectral radius is 0.0364. Thus, in this case the periodic sequence cyclic in $\{4,2,2\}$ guarantees exponential stability, resulting in a much simpler control. Nevertheless, the state dependent control policies in (25) and in Remark 3 provide much better performances, see Figure 1 where $x_{0}=(1,1,0,-1)$.

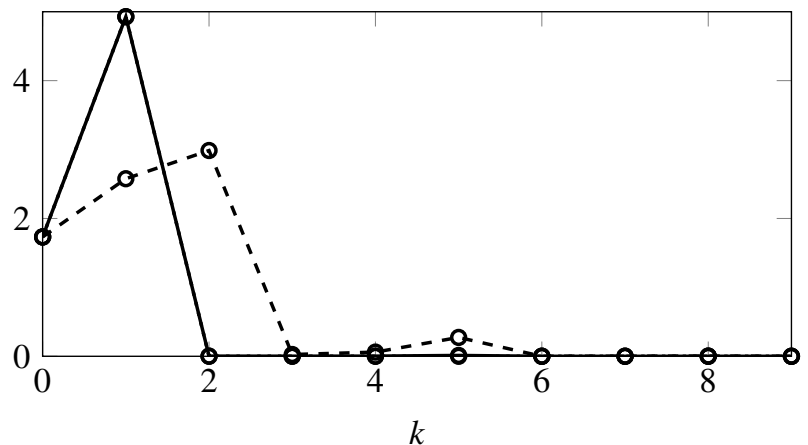

Fig. 1. Evolutions of $\|x\|_{2}$ with control (25) and min-switching of Remark 3 in solid, periodic control (29) in dashed.

Finally, as $A_{4}$ is already Schur-Cohn, with 4 eigenvalues in 0.5 , we define a new $A_{4}$ multiplying it by 2.5 . All the eigenvalues of $A_{4}$ are now in 1.25. The evolutions of the Euclidean norm of the state, for $x_{0}=(1,1,0,-1)$, under the obtained controls are depicted in Figure 2.

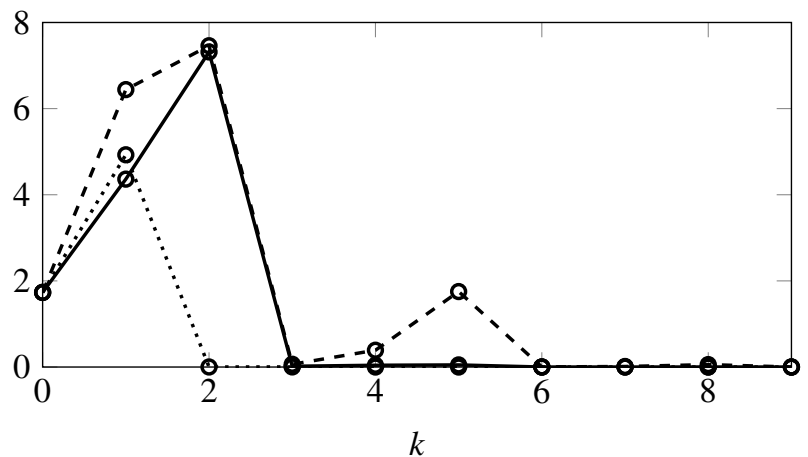

Fig. 2. Evolutions of $\|x\|_{2}$ with control (25) in solid, periodic control (29) in dashed; min-switching of Remark 3 in dotted line.

\section{Conclusion}

In this paper we presented a necessary and sufficient condition for stabilizability of periodic stabilizable discrete-time switched linear systems by co-designing both the switching law and the feedback gain. The condition is in form of an LMI, whose dimension depends combinatorially on the considered horizon, and can be used to maximize the convergence rate. The method is compared with results from the literature in terms of computation, conservatism and performance.

\section{References}

[1] D. Antunes and W. P. M. H. Heemels. Linear quadratic regulation of switched systems using informed policies. IEEE Transactions on Automatic Control, PP(99):1-1, 2016.

[2] F. Blanchini and C. Savorgnan. Stabilizability of switched linear systems does not imply the existence of convex Lyapunov functions. Automatica, 44:1166 - 1170, 2008.

[3] S. Boyd, L. El Ghaoui, E. Feron, and V. Balakrishnan. Linear Matrix Inequalities in system and control theory. SIAM Studies in Applied Mathematics, Philadelphia, 1994.

[4] J. Daafouz, P. Riedinger, and C. Iung. Stability analysis and control synthesis for switched systems: A switched Lyapunov function approach. IEEE Transactions on Automatic Control, 47:1883-1887, 2002.

[5] G. S. Deaecto, J. C. Geromel, and J. Daafouz. Dynamic output feedback $\mathrm{H}_{\infty}$ control of switched linear systems. Automatica, 47(8):1713-1720, 2011.

[6] G. S. Deaecto, M. Souza, and J. C. Geromel. Discrete-time switched linear systems state feedback design with application to networked control. IEEE Transactions on Automatic Control, 60(3):877-881, 2015.

[7] M. Fiacchini, A. Girard, and M. Jungers. On the stabilizability of discrete-time switched linear systems: Novel conditions and comparisons. IEEE Transactions on Automatic Control, 61(5):11811193, 2016.

[8] M. Fiacchini and M. Jungers. Necessary and sufficient condition for stabilizability of discrete-time linear switched systems: A set-theory approach. Automatica, 50(1):75 - 83, 2014.

[9] J. C. Geromel and P. Colaneri. Stability and stabilization of discretetime switched systems. International Journal of Control, 79(7):719_ 728, July 2006.

[10] W. P. M. H. Heemels, A. Kundu, and J. Daafouz. On LyapunovMetzler inequalities and S-procedure characterisations for the stabilisation of switched linear systems. IEEE Transactions on Automatic Control, PP(99):1-1, 2016.

[11] R. M. Jungers. The Joint Spectral Radius: Theory and Applications. Springer-Verlag., Berlin Heidelberg, June 2009.

[12] D. Liberzon. Switching in Systems and Control. Birkhäuser, Boston, MA, 2003.

[13] H. Lin and P. J. Antsaklis. Stability and stabilizability of switched linear systems: a survey of recent results. IEEE Transaction on Automatic Control, 54(2):308-322, 2009.

[14] A. P. Molchanov and Y. S. Pyatnitskiy. Criteria of asymptotic stability of differential and difference inclusions encounterd in control theory. Systems \& Control Letters, 13:59-64, 1989.

[15] G. Pipeleers, B. Demeulenaere, J. Swevers, and L. Vandenberghe. Extended LMI characterizations for stability and performance of linear systems. Systems \& Control Letters, 58(7):510-518, 2009.

[16] Z. Sun and S. S. Ge. Stability Theory of Switched Dynamical Systems. Springer, 2011.

[17] W. Zhang, A. Abate, J. Hu, and M. P. Vitus. Exponential stabilization of discrete-time switched linear systems. Automatica, 45(11):25262536, 2009.

[18] W. Zhang, J. Hu, and A. Abate. Infinite-horizon switched lqr problems in discrete time: a suboptimal algorithm with performance analysis. IEEE Transactions on Automatic Control, 57(7):1815-1821, 2012. 